Research Article

\title{
Lower Bounds on the Entire Zagreb Indices of Trees
}

\author{
Liang Luo, ${ }^{1,2}$ Nasrin Dehgardi $\left(\mathbb{D},{ }^{3} \text { and Asfand Fahad }{ }^{4}\right)^{4}$ \\ ${ }^{1}$ Key Laboratory of High Performance Ship Technology, Wuhan University of Technology, Ministry of Education, \\ Wuhan 430070, China \\ ${ }^{2}$ School of Transportation, Wuhan University of Technology, Wuhan 430070, China \\ ${ }^{3}$ Department of Mathematics and Computer Science, Sirjan University of Technology, Sirjan, Iran \\ ${ }^{4}$ Department of Mathematics, COMSATS University Islamabad, Vehari Campus, Vehari 61100, Pakistan
}

Correspondence should be addressed to Nasrin Dehgardi; n.dehgardi@sirjantech.ac.ir

Received 16 February 2020; Revised 22 March 2020; Accepted 4 April 2020; Published 27 April 2020

Academic Editor: Youssef N. Raffoul

Copyright (c) 2020 Liang Luo et al. This is an open access article distributed under the Creative Commons Attribution License, which permits unrestricted use, distribution, and reproduction in any medium, provided the original work is properly cited.

For a (molecular) graph $G$, the first and the second entire Zagreb indices are defined by the formulas $M_{1}^{\varepsilon}(G)=\sum_{x \in V(G) \cup E(G)} d(x)^{2}$ and $M_{2}^{\varepsilon}(G)=\sum_{x \text { is either adjacent or incident to } y} d(x) d(y)$ in which $d(x)$ represents the degree of a vertex or an edge $x$. In the current manuscript, we establish some lower bounds on the first and the second entire Zagreb indices and determine the extremal trees which achieve these bounds.

\section{Introduction}

In the modern era, certain graph invariants present significant applications not only in mathematics but also in other branches of science such as characterization of a molecular structure in computational chemistry and determination of the energies of $\pi$-electrons in the chemical physics. These invariants may associate a numeric number, a polynomial, a matrix, a sequence of numbers, or a drawing to a graph (or an equivalent object such as a molecular structure). Such values are either identical or possess similar trends for isomorphic graphs. One such revolutionary example of these invariants is a topological index (TI) of the graphs (or equivalent objects), which is sensitive to symmetry, size, bonding pattern, and the shape in a graph. Consequently, the study of the TIs contributes greatly to the quantitative characterizations of the objects (which are equivalent to graphs) in several branches of science. Therefore, the evaluation and the analysis of TIs of graphs are modern trends of research having significant importance in many branches of science, for example, in nanotechnology and theoretical chemistry. A prediction on bioactivity of molecular structures (chemical compounds) in QSAR/QSPR studies can be made on the basis of these graph invariants. Thus, the analysis of the lower/upper bounds of these invariants may possess a significant impact in mathematics and in the prediction of bioactivity. For a comprehensive literature review, the readers are referred to [1-4].

Throughout this manuscript, we use the standard notations from the field of graph theory. We consider the simple and connected graph $G$, and denote a vertex set by $V=V(G)$, edge set by $E=E(G)$, the cardinality of $V$ by $n=n(G)$, and the cardinality of $E$ by $m=m(G)$. Furthermore, we denote the degree of a vertex $v$ by $d_{G}(v)=$ $d(v)$ (degree of an edge $e$ by $d_{G}(e)=d(e)$ ), minimum and maximum degrees by $\delta=\delta(G)$ and $\Delta=\Delta(G)$, respectively, and the open neighborhood of $v$ in $G$ by $N(v / G)$. Lastly, $\ell(v)$ denotes the number of leaves adjacent to the vertex $v$, $D(v)$ denotes the set of descendants of $v$, and depth $(v)$ is the largest distance from $v$ to a vertex in $D(v)$. For the notions and notations not given here, we refer the readers [5].

For a simple and connected graph $G$, the first and the second Zagreb indices are defined in terms of the degrees of the vertices by the formulas $M_{1}(G)=\sum_{v \in V(G)} d(v)^{2}$ and $M_{2}(G)=\sum_{u v \in V(G)} d(u) d(v)$, see $[6,7]$. The Zagreb indices are among the oldest TIs and have been studied extensively. The development of the study of these indices along with their applications can be seen in the surveys [8-11]. 
In a recent study related to these TIs, several variants of these TIs have been introduced and their properties and applications have been analysed. For example, Zagreb coindices [12], graph operations [13], reformulated Zagreb indices $[14,15]$, Zagreb hyperindex of graph operations, Zagreb hyperindex and its coindices [16, 17], multiplicative Zagreb indices [18, 19], general Zagreb index [20], multiplicative sum Zagreb index [21, 22], multiplicative Zagreb coindices [23], general first Zagreb index [24], and the First Zagreb index [25]. In [26], an upper bound on the first Zagreb index and coindex in trees was established. In [24], the general first Zagreb index has been expressed in terms of a star sequence. Behtoei [27] studied bounds and relations of the general first Zagreb index and modified the results proved in [24]. For further aspects of the topological descriptors and their applications see $[25,28,29]$.

Recently, Alwardi et al. [30] extended the concept of Zagreb indices to the vertex and edge degrees, conceiving the so-called entire Zagreb indices. For a simple and connected graph $G$, the formulas $M_{1}^{\varepsilon}(G)=\sum_{x \in V(G) \cup E(G)} d(x)^{2}$ and $M_{2}^{\varepsilon}(G)=\sum x$ is either adjacentor incident to $y d(x) d(y)$ define the first and the second entire Zagreb indices. Moreover, the basic properties including bounds in terms of Zagreb indices, order, and the size of the underlying graph $G$ were established. In [31], the relationship between the entire Zagreb indices and reformulated Zagreb indices has been developed. Along with this, several inequalities related to these indices involving different graphs have been proved. In this manuscript, we intend to establish some lower bounds on the first and the second entire Zagreb indices and determine those external trees which achieve these bounds. The following theorem providing the exact values of the first and the second entire Zagreb indices for the path $P_{n}$ (with $n \geq 3$ ) will be useful to determine the lower bounds for trees.

Theorem 1 (see [30]). For any path $P_{n}$ with $n \geq 3, M_{1}^{\varepsilon}\left(P_{n}\right)=$ $8(n-2) \quad$ and $M_{2}^{\varepsilon}\left(P_{n}\right)=11$ when $n=3$ and $M_{2}^{\varepsilon}\left(P_{n}\right)=2(8 n-19)$ when $n \geq 4$.

Before proceeding further, we include a remark which will be helpful to establish a lower bound for any graph $G$ by using a lower bound for a tree.

Remark 1. Let $G$ be a graph. Then, for every edge $e \notin E(G)$,
(a) $M_{1}^{\varepsilon}(G+e)>M_{1}^{\varepsilon}(G)$.
(b) $M_{2}^{\varepsilon}(G+e)>M_{2}^{\varepsilon}(G)$.

\section{Main Results}

Throughout this section, $T$ denotes a rooted tree with root $\omega$, where $\omega$ is a vertex of a maximum degree and $N(\omega)=\left\{w_{1}, w_{2}, \ldots, w_{\Delta}\right\}$. Firstly, for a tree with some useful conditions, we prove existence of another tree of the same order and the same maximum degree which provide lower bounds on the first and second entire Zagreb indices (Figures 1-3).

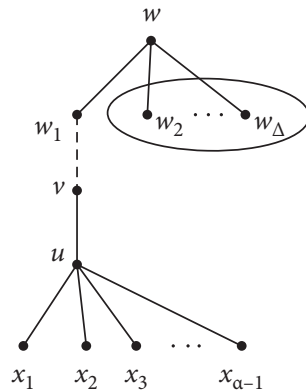

$T$

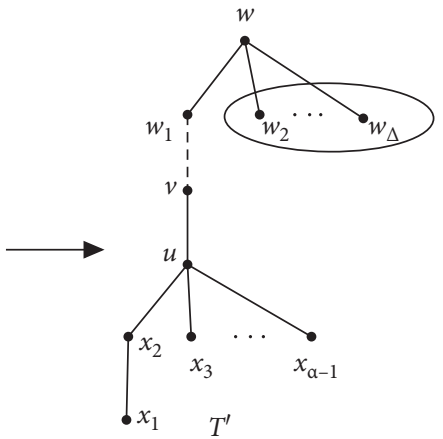

Figure 1: Case 1.
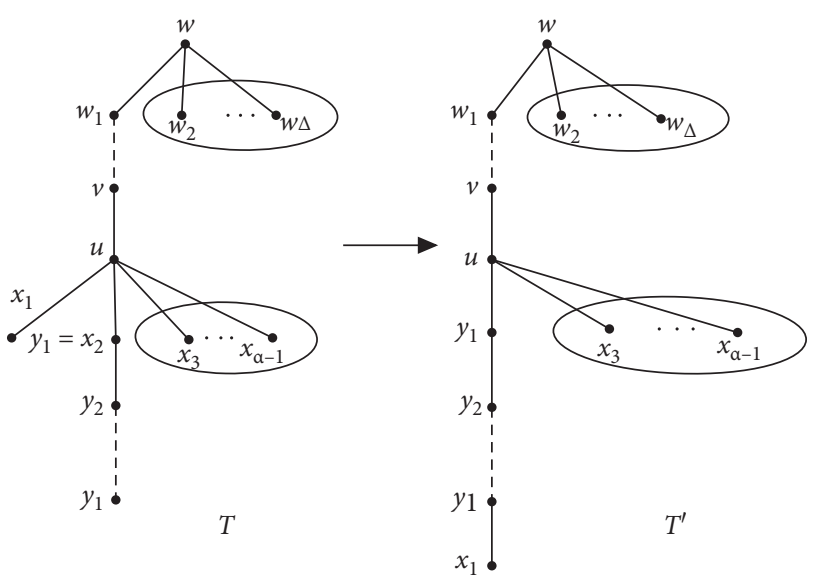

Figure 2: Case 2.
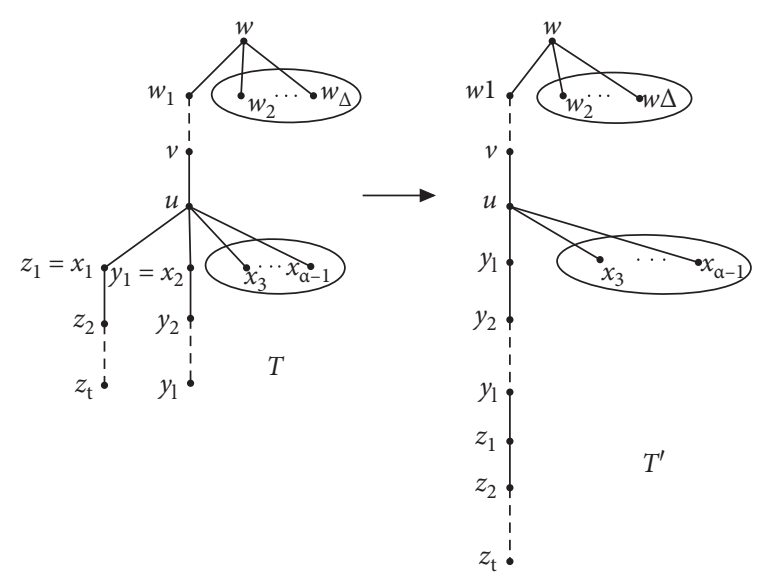

FIgure 3: Case 3.

Lemma 1. Let $T$ be a tree of order $n$ with a maximum degree $\Delta$. If $T$ has a vertex $u$ of degree at least three in maximum distance from $\omega$, then there is a tree $T$ of order $n$ with a maximum degree $\Delta$ such that $M_{1}^{\varepsilon}\left(T^{\prime}\right)<M_{1}^{\varepsilon}(T)$ and $M_{2}^{\varepsilon}\left(T^{\prime}\right)<M_{2}^{\varepsilon}(T)$.

Proof. Let $u \neq \omega$ be an end-stem of $T$ with $d(u)=\alpha \geq 3$ and let $N_{T}(u)=\left\{x_{1}, x_{2}, \ldots, x_{\alpha-1}, x_{\alpha}=v\right\}$, where $v$ is the parent of $u$. We consider the following cases. 
Case 1. All neighbors of $u$ except $v$ are leaves.

Let $T^{\prime}$ be the tree obtained from $T-\left\{x_{1}\right\}$ by attaching the path $x_{1} x_{2}$. Then, we have

$$
\begin{aligned}
& M_{1}^{\varepsilon}(T)-M_{1}^{\varepsilon}\left(T^{\prime}\right)=\sum_{x \in V(T) \cup E(T)} d(x)^{2}-\sum_{x \in V\left(T^{\prime}\right) \cup E\left(T^{\prime}\right)} d(x)^{2} \\
& =\sum_{i=1}^{\alpha-1} d_{T}\left(u x_{i}\right)^{2}+d_{T}\left(x_{2}\right)^{2}+d_{T}(u)^{2}+d_{T}(u v)^{2} \\
& -\sum_{i=3}^{\alpha-1}\left(d_{T}\left(u x_{i}\right)-1\right)^{2}-\left(d_{T}(u)-1\right)^{2}-\left(d_{T}(u v)-1\right)^{2} \\
& -d_{T^{\prime}}\left(x_{2}\right)^{2}-d_{T^{\prime}}\left(x_{1} x_{2}\right)^{2}-d_{T^{\prime}}\left(u x_{2}\right)^{2} \\
& =\sum_{i=3}^{\alpha-1}\left[d_{T}\left(u x_{i}\right)^{2}-\left(d_{T}\left(u x_{i}\right)-1\right)^{2}\right]+\alpha^{2}-4+d_{T}(u v)^{2}-\left(d_{T}(u v)-1\right)^{2}+ \\
& >\alpha^{2}-4 \geq 0 \text {, } \\
& M_{2}^{\varepsilon}(T)-M_{2}^{\varepsilon}\left(T^{\prime}\right)=\sum_{x \text { is either adjacent or incident to } y} d_{T}(x) d_{T}(y)-\sum_{x \text { is either adjacent or incident to } y} d_{T^{\prime}}(x) d_{T^{\prime}}(y) \\
& =\sum_{i=1}^{\alpha}\left[d_{T}\left(x_{i}\right) d_{T}(u)+d_{T}\left(x_{i}\right) d_{T}\left(u x_{i}\right)+d_{T}(u) d_{T}\left(u x_{i}\right)\right]+\sum_{1 \leq i j \leq \alpha} d_{T}\left(u x_{i}\right) d_{T}\left(u x_{j}\right)+\sum_{w \in N_{T}(v)} d_{T}(u v) d_{T}(v w) \\
& -\sum_{i=3}^{\alpha}\left[d_{T}\left(x_{i}\right)\left(d_{T}(u)-1\right)+d_{T}\left(x_{i}\right)\left(d_{T}\left(u x_{i}\right)-1\right)+\left(d_{T}(u)-1\right)\left(d_{T}\left(u x_{i}\right)-1\right)\right] \\
& +\sum_{3 \leq i<j \leq \alpha}\left(d_{T}\left(u x_{i}\right)-1\right)\left(d_{T}\left(u x_{j}\right)-1\right)+\sum_{w \in N_{T}(v)}\left(d_{T}(u v)-1\right) d_{T}(v w) \\
& -\sum_{3 \leq i \leq \alpha}\left[\left(d_{T}\left(x_{2} u\right)+1\right)\left(d_{T}\left(x_{i} u\right)-1\right)-d_{T^{\prime}}\left(x_{1}\right) d_{T^{\prime}}\left(x_{2}\right)-d_{T^{\prime}}\left(x_{1}\right) d_{T^{\prime}}\left(x_{1} x_{2}\right)\right. \\
& -d_{T^{\prime}}\left(x_{2}\right) d_{T^{\prime}}\left(x_{1} x_{2}\right)-d_{T^{\prime}}\left(x_{1} x_{2}\right)\left(d_{T}\left(u x_{2}\right)+1\right)-d_{T}\left(x_{2}\right)\left(d_{T}\left(u x_{2}\right)+1\right)-\left(d_{T}(u)-1\right)\left(d_{T}\left(u x_{2}\right)+1\right) \\
& -d_{T}^{\prime}\left(x_{2}\right)\left(d_{T}(u)-1\right) \\
& \geq 2[\alpha+\alpha-1+\alpha(\alpha-1)]+(\alpha-1)^{2}[(\alpha-2)+(\alpha-3)]+2(\alpha-1) d_{T}(u v)-(\alpha-1)(\alpha-2)(\alpha-3)-(\alpha-1) \\
& -2(\alpha-1)-(\alpha-1)\left(d_{T}(u v)-1\right)-\alpha(\alpha-1) \\
& -2(\alpha-1)-5>\alpha^{3}-2 \alpha^{2}-3>0 \text {. }
\end{aligned}
$$

Case 2. When $u$ is adjacent to a leaf $x_{1}, u y_{1} y_{2} \ldots y_{l}$ is a path in $T$ for $l \geq 2$ and $x_{2}=y_{1}$.

Let $T^{\prime}$ be the tree obtained from $T-\left\{x_{1}\right\}$ by attaching the path $y_{l} x_{1}$. Then, 


$$
\begin{aligned}
M_{1}^{\varepsilon}(T)-M_{1}^{\varepsilon}\left(T^{\prime}\right)= & \sum_{x \in V(T) \cup E(T)} d(x)^{2}-\sum_{x \in V\left(T^{\prime}\right) \cup E\left(T^{\prime}\right)} d(x)^{2}=\sum_{i=2}^{\alpha} d_{T}\left(u x_{i}\right)^{2}+d_{T}(u)^{2}+d_{T}\left(u x_{1}\right)^{2}+d_{T}\left(y_{l}\right)^{2}+d_{T}\left(y_{l} y_{l-1}\right)^{2} \\
& -\sum_{i=2}^{\alpha}\left(d_{T}\left(u x_{i}\right)-1\right)^{2}-\left(d_{T}(u)-1\right)^{2}-\left(d_{T}\left(y_{l}\right)+1\right)^{2}-d_{T^{\prime}}\left(x_{1} y_{l}\right)^{2}-\left(d_{T}\left(y_{l} y_{l-1}\right)+1\right)^{2}>(\alpha-1)^{2}+\alpha^{2} \\
& +2-9-(\alpha-1)^{2}=\alpha^{2}-7>0,
\end{aligned}
$$

$$
\begin{aligned}
& M_{2}^{\varepsilon}(T)-M_{2}^{\varepsilon}\left(T^{\prime}\right)= \sum_{x \text { is either adjacent or incident to } y} d_{T}(x) d_{T}(y)-\sum_{x \text { is either adjacent or incident to } y} d_{T^{\prime}}(x) d_{T^{\prime}}(y) \\
&= \sum_{i=1}^{\alpha}\left[d_{T}\left(x_{i}\right) d_{T}(u)+d_{T}\left(x_{i}\right) d_{T}\left(u x_{i}\right)+d_{T}(u) d_{T}\left(u x_{i}\right)\right]+\sum_{1 \leq i<j \leq \alpha} d_{T}\left(u x_{i}\right) d_{T}\left(u x_{j}\right) \\
&+\sum_{w \in N_{T}(v)} d_{T}(u v) d_{T}(v w)+d_{T}\left(y_{l}\right) d_{T}\left(y_{l-1}\right)+d_{T}\left(y_{l}\right) d_{T}\left(y_{l} y_{l-1}\right)+d_{T}\left(y_{l-1}\right) d_{T}\left(y_{l} y_{l-1}\right)+d_{T}\left(y_{l-1} y_{l-2}\right) \\
& d_{T}\left(y_{l} y_{l-1}\right)-\sum_{i=2}^{\alpha}\left[d_{T}\left(x_{i}\right)\left(d_{T}(u)-1\right)+d_{T}\left(x_{i}\right)\left(d_{T}\left(u x_{i}\right)-1\right)+\left(d_{T}(u)-1\right)\left(d_{T}\left(u x_{i}\right)-1\right)\right] \\
&-\sum_{2 \leq i<j \leq \alpha}\left(d_{T}\left(u x_{i}\right)-1\right)\left(d_{T}\left(u x_{j}\right)-1\right)-\sum_{w \in N_{T}(v)}\left(d_{T}(u v)-1\right) d_{T}(v w)-d_{T^{\prime}}\left(x_{1}\right) d_{T^{\prime}}\left(y_{l}\right) \\
&-d_{T^{\prime}}\left(x_{1}\right) d_{T^{\prime}}\left(x_{1} y_{l}\right) \\
&-d_{T^{\prime}}\left(y_{l-1} y_{l-2}\right) d_{T^{\prime}}\left(y_{l} y_{l-1}\right)-d_{T^{\prime}}^{2}\left(y_{l}\right) d_{T^{\prime}}^{2}\left(y_{l-1}\right) \\
& d_{T^{\prime}}\left(y_{l}\right) d_{T^{\prime}}\left(x_{1} y_{l}\right)-d_{T^{\prime}}\left(x_{1} y_{l}\right) d_{T^{\prime}}\left(y_{l} y_{l-1}\right)-d_{T^{\prime}}\left(y_{l}\right) d_{T^{\prime}}\left(y_{l} y_{l-1}\right)-d_{T^{\prime}}\left(y_{l-1}\right) d_{T^{\prime}}\left(y_{l} y_{l-1}\right) \\
& \geq \alpha^{3}-2 \alpha^{2}+5 \alpha-17-d_{T}\left(y_{l-1} y_{l-2}\right)>0 .
\end{aligned}
$$

Case 3. When $u$ is not a stem, $u z_{1} z_{2}, \ldots, z_{t}$, and $u y_{1} y_{2}, \ldots, y_{l},(l, t \geq 2)$ are two paths in $T$ such that $x_{1}=z_{1}$ and $x_{2}=y_{1}$.
Let $T^{\prime}$ be the tree obtained from $T-\left\{z_{1}\right\}$ by attaching the path $y_{l} z_{1}$. Therefore, we have

$$
\begin{aligned}
M_{1}^{\varepsilon}(T)-M_{1}^{\varepsilon}\left(T^{\prime}\right)= & \sum_{x \in V(T) \cup E(T)} d(x)^{2}-\sum_{x \in V\left(T^{\prime}\right) \cup E\left(T^{\prime}\right)} d(x)^{2} \\
= & \sum_{i=2}^{\alpha} d_{T}\left(u x_{i}\right)^{2}+d_{T}(u)^{2}+d_{T}\left(u x_{1}\right)^{2}+d_{T}\left(y_{l}\right)^{2}+d_{T}\left(y_{l} y_{l-1}\right)^{2} \\
& -\sum_{i=2}^{\alpha}\left(d_{T}\left(u x_{i}\right)-1\right)^{2}-\left(d_{T}(u)-1\right)^{2} \\
& -\left(d_{T}\left(y_{l}\right)+1\right)^{2}-d_{T^{\prime}}\left(x_{1} y_{l}\right)^{2}-\left(d_{T}\left(y_{l} y_{l-1}\right)+1\right)^{2} \\
> & 2 \alpha^{2}+2-12-(\alpha-1)^{2} \\
= & \alpha^{2}+2 \alpha-11>0, \quad \sum_{x} d_{T}(x) d_{T}(y)-\sum_{T^{\prime}}(x) d_{T^{\prime}}(y) \\
M_{2}^{\varepsilon}(T)-M_{2}^{\varepsilon}\left(T^{\prime}\right)= & \sum_{x \text { is either adjacent or incident to } y} \text { adjacent or incident to } y \quad \sum_{i=1}^{\alpha}\left[d_{T}\left(x_{i}\right) d_{T}(u)+d_{T}\left(x_{i}\right) d_{T}\left(u x_{i}\right)+d_{T}(u) d_{T}\left(u x_{i}\right)\right]+d_{T}\left(z_{1} z_{2}\right) d_{T}\left(u z_{1}\right)
\end{aligned}
$$




$$
\begin{aligned}
& +\sum_{1 \leq i<j \leq \alpha} d_{T}\left(u x_{i}\right) d_{T}\left(u x_{j}\right)+\sum_{w \in N_{T}(v)} d_{T}(u v) d_{T}(v w)+d_{T}\left(y_{l}\right) d_{T}\left(y_{l-1}\right)+d_{T}\left(y_{l}\right) \\
& \quad \cdot d_{T}\left(y_{l} y_{l-1}\right)+d_{T}\left(y_{l-1}\right) d_{T}\left(y_{l} y_{l-1}\right)+d_{T}\left(y_{l-1} y_{l-2}\right) d_{T}\left(y_{l} y_{l-1}\right) \\
& \quad-\sum_{i=2}^{\alpha}\left[d_{T}\left(x_{i}\right)\left(d_{T}(u)-1\right)+d_{T}\left(x_{i}\right)\left(d_{T}\left(u x_{i}\right)-1\right)+\left(d_{T}(u)-1\right)\left(d_{T}\left(u x_{i}\right)-1\right)\right] \sum_{2 \leq i<j \leq \alpha}\left(d_{T}\left(u x_{i}\right)-1\right)\left(d_{T}\left(u x_{j}\right)-1\right) \\
& \quad \cdot \sum_{w \in N_{T}(v)}\left(d_{T}(u v)-1\right) d_{T}(v w)-d_{T^{\prime}}\left(z_{1}\right) d_{T^{\prime}}\left(y_{l}\right)-d_{T^{\prime}}\left(z_{1}\right) d_{T^{\prime}}\left(z_{1} y_{l}\right)-d_{T^{\prime}}\left(y_{l-1} y_{l-2}\right) d_{T^{\prime}}\left(y_{l} y_{l-1}\right) \\
& \quad-d_{T^{\prime}}\left(z_{1} y_{l}\right) d_{T^{\prime}}\left(z_{1} z_{2}\right)-d_{T^{\prime}}\left(y_{l}\right) \\
& \quad d_{T^{\prime}}\left(z_{1} y_{l}\right)-d_{T^{\prime}}\left(z_{1} y_{l}\right) d_{T^{\prime}}\left(y_{l} y_{l-1}\right)-d_{T^{\prime}}\left(y_{l}\right) d_{T^{\prime}}\left(y_{l} y_{l-1}\right)-d_{T^{\prime}}\left(y_{l-1}\right) d_{T^{\prime}}\left(y_{l} y_{l-1}\right)-d_{T^{\prime}}\left(y_{l}\right) d_{T^{\prime}}\left(y_{l-1}\right) \\
& \geq \alpha^{3}+5 \alpha-29-d_{T}\left(y_{l-1} y_{l-2}\right)>0,
\end{aligned}
$$

which completes the proof.

Before proceeding further, we recall some useful definitions from the literature. A spider is a tree with at most one vertex of degree more than 2 , which is called the center of a spider. In case, there is no such vertex, then any vertex may be the center. A path from the center to a vertex of degree 1 is called a leg of a spider. Thus, a star with $k$ edges is a spider of $k$ legs, each having length 1 , and a path is a spider with 1 or 2 legs. By Lemma 1 we have that between all trees of order $n$ with a maximum degree $\Delta$, spiders have the minimum first and second entire Zagreb indices. Next, we determine the spiders having the minimum first and the second entire
Zagreb indices. If $\Delta=2$ then $T=P_{n}$. Now, we prove the following important lemma.

Lemma 2. Let $T$ be a spider of order $n$ with $p \geq 3$ legs. If $T$ has two legs of length at least 2 , then there is a spider $T^{\prime}$ of order $n$ with $p$ legs such that $M_{1}^{\varepsilon}\left(T^{\prime}\right)<M_{1}^{\varepsilon}(T)$ and $M_{2}^{\varepsilon}\left(T^{\prime}\right)<M_{2}^{\varepsilon}(T)$.

Proof. Let $\omega$ be the center of $T$ and let $\omega x_{1} x_{2}, \ldots, x_{t}$ and $\omega y_{1} y_{2}, \ldots, y_{l},(l, t \geq 2)$ be the two legs of length at least three in $T$. Let $T^{\prime}$ be the tree obtained from $T-\left\{x_{1} x_{2}\right\}$ by attaching the path $y_{l} x_{2}$. Then,

$$
\begin{aligned}
M_{1}^{\varepsilon}(T)-M_{1}^{\varepsilon}\left(T^{\prime}\right)= & \sum_{x \in V(T) \cup E(T)} d(x)^{2}-\sum_{x \in V\left(T^{\prime}\right) \cup E\left(T^{\prime}\right)} d(x)^{2} \geq d_{T}\left(x_{1}\right)^{2}+d_{T}\left(x_{1} x_{2}\right)^{2}+d_{T}\left(x_{1} w\right)^{2}+d_{T}\left(y_{l}\right)^{2} \\
& +d_{T}\left(y_{l-1} y_{l}\right)^{2}-d_{T^{\prime}}\left(x_{1}\right)^{2}-d_{T^{\prime}}\left(x_{1} w\right)^{2}-d_{T^{\prime}}\left(y_{l}\right)^{2}-d_{T^{\prime}}\left(y_{l-1} y_{l}\right)^{2}-d_{T^{\prime}}\left(x_{2} y_{l}\right)^{2} \\
= & 2 \Delta-4>0 .
\end{aligned}
$$

Now, we show that $M_{2}^{\varepsilon}\left(T^{\prime)<M_{2}^{\varepsilon}(T)}\right.$. Firstly, let $l=t=2$, then

$$
\begin{aligned}
M_{2}^{\varepsilon}(T)-M_{2}^{\varepsilon}\left(T^{\prime}\right)= & \sum_{x \text { is either adjacent or incident to } y} d_{T}(x) d_{T}(y)-\sum_{x \text { is either adjacent or incident to } y} d_{T^{\prime}}(x) d_{T^{\prime}}(y) \\
\geq & d_{T}\left(x_{1}\right) d_{T}\left(x_{2}\right)+d_{T}\left(x_{2}\right) d_{T}\left(x_{1} x_{2}\right)+d_{T}\left(x_{1}\right) d_{T}\left(x_{1} x_{2}\right)+d_{T}\left(x_{1} x_{2}\right) \\
& \cdot d_{T}\left(x_{1} w\right)+d_{T}\left(x_{1}\right) d_{T}\left(x_{1} w\right)+d_{T}\left(x_{1}\right) d_{T}(w)+d_{T}\left(y_{1}\right) d_{T}\left(y_{1} y_{2}\right)+d_{T}\left(y_{2}\right) d_{T}\left(y_{1} y_{2}\right) \\
& +d_{T}\left(y_{1}\right) d_{T}\left(y_{2}\right)+d_{T}\left(x_{1} w\right) \\
& \cdot d_{T}\left(w y_{1}\right)+d_{T}\left(y_{1} w\right) d_{T}\left(y_{1} y_{2}\right)+d_{T}(w) d_{T}\left(x_{1} w\right)-d_{T^{\prime}}\left(x_{1} w\right) d_{T}\left(y_{1} w\right)-d_{T^{\prime}}\left(x_{1}\right) d_{T}\left(x_{1} w\right) \\
& -d_{T^{\prime}}\left(x_{1}\right) d_{T}(w)-d_{T^{\prime}}(w) \\
& \cdot d_{T}\left(x_{1} w\right)-d_{T^{\prime}}\left(x_{2}\right) d_{T^{\prime}}\left(x_{2} y_{2}\right)-d_{T^{\prime}}\left(x_{2}\right) d_{T^{\prime}}\left(y_{2}\right)-d_{T^{\prime}}\left(x_{2} y_{2}\right) d_{T^{\prime}}\left(y_{2}\right)-d_{T^{\prime}}\left(y_{1}\right) d_{T^{\prime}}\left(y_{2}\right) \\
& -d_{T^{\prime}}\left(y_{1} y_{2}\right) d_{T^{\prime}}\left(x_{2} y_{2}\right)-d_{T^{\prime}}\left(y_{1}\right) d_{T^{\prime}}\left(y_{1} y_{2}\right)-d_{T^{\prime}}\left(y_{1} y_{2}\right) d_{T^{\prime}}\left(y_{2}\right)-d_{T^{\prime}}\left(y_{1} y_{2}\right) d_{T^{\prime}}\left(w y_{1}\right) \\
= & 4 \Delta-8>0 .
\end{aligned}
$$

If $t=2$ and $l \geq 3$, then 


$$
\begin{aligned}
M_{2}^{\varepsilon}(T)-M_{2}^{\varepsilon}\left(T^{\prime}\right)= & \sum_{x \text { is either adjacent or incident to } y} d_{T}(x) d_{T}(y)-\sum_{x \text { is either adjacent or incident to } y} d_{T^{\prime}}(x) d_{T^{\prime}}(y) \\
\geq & d_{T}\left(x_{1}\right) d_{T}\left(x_{2}\right)+d_{T}\left(x_{2}\right) d_{T}\left(x_{1} x_{2}\right)+d_{T}\left(x_{1}\right) d_{T}\left(x_{1} x_{2}\right)+d_{T}\left(x_{1} x_{2}\right) d_{T}\left(x_{1} w\right)+d_{T}\left(x_{1}\right) \\
& \cdot d_{T}\left(x_{1} w\right)+d_{T}\left(x_{1}\right) d_{T}(w)+d_{T}\left(y_{l-1}\right) d_{T}\left(y_{l-1} y_{l}\right)+d_{T}\left(y_{l}\right) \\
& \cdot d_{T}\left(y_{l-1} y_{l}\right)+d_{T}\left(y_{l-1}\right) d_{T}\left(y_{l}\right)+d_{T}\left(y_{l} y_{l-1}\right) d_{T}\left(y_{l-1} y_{l-2}\right)+d_{T}\left(x_{1} w\right) d_{T}\left(w y_{1}\right)+d_{T}(w) \\
& \cdot d_{T}\left(x_{1} w\right)-d_{T^{\prime}}\left(x_{1} w\right) d_{T}\left(y_{1} w\right)-d_{T^{\prime}}\left(x_{1}\right) d_{T}\left(x_{1} w\right)-d_{T^{\prime}}\left(x_{1}\right) \\
& \cdot d_{T}\left(x_{1} w\right)-d_{T^{\prime}}\left(x_{1}\right) d_{T}(w)-d_{T^{\prime}}(w) d_{T}\left(x_{1} w\right)-d_{T^{\prime}}\left(x_{2}\right) d_{T}\left(x_{2} y_{l}\right)-d_{T^{\prime}}\left(x_{2}\right) d_{T^{\prime}}\left(y_{l}\right) \\
& -d_{T^{\prime}}\left(x_{2} y_{l}\right) d_{T^{\prime}}\left(y_{l}\right)-d_{T^{\prime}}\left(y_{l-1}\right) \\
& \cdot d_{T^{\prime}}\left(y_{l}\right)-d_{T^{\prime}}\left(y_{l-1} y_{l}\right) d_{T^{\prime}}\left(x_{2} y_{l}\right)-d_{T^{\prime}}\left(y_{l-1}\right) d_{T^{\prime}}\left(y_{l-1} y_{l}\right)-d_{T^{\prime}}\left(y_{l-1} y_{l}\right) d_{T^{\prime}}\left(y_{l}\right) \\
& -d_{T^{\prime}}\left(y_{l-1} y_{l}\right) d_{T^{\prime}}\left(y_{l-1} y_{l-2}\right) \\
= & 5 \Delta-10>0 .
\end{aligned}
$$

Finally, let $l, t \geq 3$, then

$$
\begin{aligned}
M_{2}^{\varepsilon}(T)-M_{2}^{\varepsilon}\left(T^{\prime}\right)= & \sum_{x \text { is either adjacent or incident to } y} d_{T}(x) d_{T}(y)-\sum_{x \text { is either adjacent or incident to } y} d_{T^{\prime}}(x) d_{T^{\prime}}(y) \\
\geq & d_{T}\left(x_{1}\right) d_{T}\left(x_{2}\right)+d_{T}\left(x_{2}\right) d_{T}\left(x_{1} x_{2}\right)+d_{T}\left(x_{1}\right) d_{T}\left(x_{1} x_{2}\right)+d_{T}\left(x_{1} x_{2}\right) d_{T}\left(x_{1} w\right)+d_{T}\left(x_{1}\right) \\
& \cdot d_{T}\left(x_{1} w\right)+d_{T}\left(x_{1}\right) d_{T}(w)+d_{T}\left(y_{l-1}\right) d_{T}\left(y_{l-1} y_{l}\right)+d_{T}\left(y_{l}\right) \\
& \cdot d_{T}\left(y_{l-1} y_{l}\right)+d_{T}\left(y_{l-1}\right) d_{T}\left(y_{l}\right)+d_{T}\left(y_{l} y_{l-1}\right) d_{T}\left(y_{l-1} y_{l-2}\right)+d_{T}\left(x_{1} w\right) \\
& \cdot d_{T}\left(w y_{1}\right)+d_{T}(w) d_{T}\left(x_{1} w\right)-d_{T^{\prime}}\left(x_{1} w\right) d_{T}\left(y_{1} w\right)-d_{T^{\prime}}\left(x_{1}\right) \\
& \cdot d_{T}\left(x_{1} w\right)-d_{T^{\prime}}\left(x_{1}\right) d_{T}(w)-d_{T^{\prime}}(w) \\
& \cdot d_{T}\left(x_{1} w\right)-d_{T^{\prime}}\left(x_{2}\right) d_{T}\left(x_{2} y_{l}\right)-d_{T^{\prime}}\left(x_{2}\right) d_{T^{\prime}}\left(y_{l}\right)-d_{T^{\prime}}\left(x_{2} y_{l}\right) d_{T^{\prime}}\left(y_{l}\right)-d_{T^{\prime}}\left(y_{l-1}\right) d_{T^{\prime}}\left(y_{l}\right) \\
& -d_{T^{\prime}}\left(y_{l-1} y_{l}\right) d_{T^{\prime}}\left(x_{2} y_{l}\right)-d_{T^{\prime}}\left(y_{l-1}\right) d_{T^{\prime}}\left(y_{l-1} y_{l}\right)-d_{T^{\prime}}\left(y_{l-1} y_{l}\right) d_{T^{\prime}}\left(y_{l}\right) \\
& -d_{T^{\prime}}\left(y_{l-1} y_{l}\right) d_{T^{\prime}}\left(y_{l-1} y_{l-2}\right)=6 \Delta-8>0
\end{aligned}
$$

which completes the proof.

In the following theorems, we prove the lower bounds on the first and the second entire Zagreb indices in terms of the maximum degree $\Delta$.

Theorem 2. For any tree $T$ of order $n \geq 3$ with a maximum degree $\Delta$, we have

(i) $M_{1}^{\varepsilon}(T) \geq \Delta^{3}-\Delta^{2}+8 n-4 \Delta-12$

whenever

$$
\Delta<n-1 \text {. }
$$

(ii) $M_{1}^{\varepsilon}(T) \geq n^{3}-4 n^{2}+7 n-4$, for $\Delta=n-1$.

Moreover, the equality corresponding to each case holds if and only if $T$ is a spider with at most one leg of length at least two.
Proof. Let $T_{1}$ be a tree of order $n \geq 3$ with a maximum degree $\Delta$ such that $M_{1}^{\varepsilon}\left(T_{1}\right)=\min \left\{M_{2}^{\varepsilon}(T) \mid T\right.$ is a tree of order $n$ with maximum degree $\Delta$ \}. Let $v$ be a vertex with a maximum degree $\Delta$ and $\operatorname{root} T_{1}$ at $v$. If $\Delta=2$, then $T$ is a path of order $n$ and the result follows by Theorem 1. Let $\Delta \geq 3$. By the choice of $T_{1}$, we deduce from Lemma 1 that $T_{1}$ is a spider with center $v$. It follows from Lemma 2 and the choice of $T_{1}$ that $T_{1}$ has at most one leg of length at least two. First let all legs of $T_{1}$ have length one. Then, $T_{1}$ is a star of order $n$ and $M_{1}^{\varepsilon}\left(T_{1}\right)=n^{3}-4 n^{2}+7 n-4$. Now, let $T_{1}$ have only one leg of length at least two. Then, $M_{2}^{\varepsilon}\left(T_{1}\right)=\Delta^{3}-\Delta^{2}+8 n-4 \Delta-12$, and this completes the proof.

Theorem 3. For any tree $T$ of order $n \geq 3$ with a maximum degree $\Delta$, we get 


$$
\begin{aligned}
& \begin{aligned}
\text { (i) } M_{2}^{\varepsilon}(T) \geq & (\Delta-1)^{2}\left[1+2 \Delta+\frac{(\Delta-1)(\Delta-2)}{2}\right]+2 \Delta^{2}-11 \Delta+16 n-29 \text { for } \Delta<n-2, \\
\text { (ii) } M_{2}^{\varepsilon}(T) \geq & (n-3)^{2}\left[2 n+\frac{(n-3)(n-4)}{2}-3\right] \\
& +(n-3)(n-2)+(n-2)^{2}+5 n-5 \text { if } \Delta=n-2, \\
\text { (iii) } M_{2}^{\varepsilon}(T) \geq & (n-1)(2 n-3)+(n-1)^{2}(n-2)+\frac{(n-1)(n-2)^{3}}{2} \text { for } \Delta=n-1 .
\end{aligned}
\end{aligned}
$$

Furthermore, the equality in each case holds if and only if $T$ is a spider with at most one leg of length at least two.

Proof. The proof is similar to the proof of Theorem 2.

By Remark 1, we obtain the following corollary.

Corollary 1. Let $G$ be a graph of order $n$ and maximum degree $\Delta$. Then,

(i) $M_{1}^{\varepsilon}(G) \geq \Delta^{3}-\Delta^{2}+8 n-4 \Delta-12$, whenever $\Delta<n-1$,

(ii) $M_{1}^{\varepsilon}(G) \geq n^{3}-4 n^{2}+7 n-4$, for $\Delta=n-1$,

(iii) $M_{2}^{\varepsilon}(G) \geq(\Delta-1)^{2}\left[1+2 \Delta+\frac{(\Delta-1)(\Delta-2)}{2}\right]+$

$$
2 \Delta^{2}-11 \Delta+16 n-29, \text { whenever } \Delta<n-2,
$$

(iv) $M_{2}^{\varepsilon}(G) \geq(n-3)^{2}\left[2 n+\frac{(n-3)(n-4)}{2}-3\right]$

$$
+(n-3)(n-2)+(n-2)^{2}+5 n-5, \text { for } \Delta=n-2 \text {, }
$$

(v) $M_{2}^{\varepsilon}(G) \geq(n-1)(2 n-3)+(n-1)^{2}(n-2)$

$$
+\frac{(n-1)(n-2)^{3}}{2}, \text { if } \Delta=n-1
$$

The equality corresponding to each case holds if and only if $G$ is a spider with at most one leg of length at least two.

\section{Data Availability}

The data used to support the findings of this study have been cited at relevant places within the text as references.

\section{Conflicts of Interest}

The authors declare no conflicts of interest.

\section{Acknowledgments}

This work was supported by Green Intelligent Inland Ship Innovation Programme. Moreover, all the authors are thankful to their respective institutes for the support during this research. Lastly, Asfand Fahad is also thankful to the Higher Education Commission of Pakistan.

\section{References}

[1] W. Gao, Z. Iqbal, M. Ishaq, A. Aslam, M. Aamir, and M. A. Binyamin, "Bounds on topological descriptors of the corona product of," IEEE Access, vol. 7, pp. 26788-26796, 2019.

[2] Z. Shao, P. Wu, X. Zhang, D. Dimitrov, and J. B. Liu, "On the maximum $\mathrm{ABC}$ index of graphs with prescribed size and without pendent vertices," IEEE Access, vol. 6, pp. 2760427616, 2018.

[3] Z. Shao, P. Wu, Y. Gao, I. Gutman, and X. Zhang, "On the maximum $\mathrm{ABC}$ index of graphs without pendent vertices," Applied Mathematics and Computation, vol. 315, pp. 298-312, 2017.

[4] J. Zheng, Z. Iqbal, A. Fahad et al., "Some eccentricity-based topological indices and polynomials of oly (ethyleneamidoamine) (PETAA) dendrimers," Processes, vol. 7, p. 433, 2019.

[5] D. B. West, Introduction to Graph Theory, Prentice-Hall, Upper Saddle River, NJ, USA, 2nd edition, 2001.

[6] I. Gutman, B. Ruščić, N. Trinajstić, and C. F. Wilcox, "Graph theory and molecular orbitals, XII. Acyclic polyenes," The Journal of Chemical Physics, vol. 62, pp. 3399-3405, 1975.

[7] I. Gutman and N. Trinajstić, "Graph theory and molecular orbitals. Total $\pi$-electron energy of alternant hydrocarbons," Chemical Physics Letters, vol. 17, pp. 535-538, 1972.

[8] B. Borovićanin, K. C. Das, B. Furtula, and I. Gutman, "Bounds for Zagreb indices," MATCH Communications in Mathematical and in Computer Chemistry, vol. 78, pp. 17-100, 2017.

[9] K. C. Das and I. Gutman, "Some properties of the second Zagreb index, MATCH Commun," MATCH Communications in Mathematical and in Computer Chemistry, vol. 52, pp. 103-112, 2004.

[10] K. C. Das and I. Gutman, "The first Zagreb index 30 years after," MATCH Communications in Mathematical and in Computer Chemistry, vol. 50, pp. 83-92, 2004. 
[11] S. Nikolić, G. Kovačević, A. Miličević, and N. Trinajstić, "The Zagreb indices 30 years after," Croatica Chemica Acta, vol. 76, pp. 113-124, 2003.

[12] I. Gutman, B. Furtula, K. Vukičević, and G. Popivoda, "On Zagreb indices and coindices," MATCH Communications in Mathematical and in Computer Chemistry, vol. 74, pp. 5-16, 2015.

[13] A. R. Ashrafi, T. Došlić, and A. Hamzeh, "The Zagreb coindices of graph operations," Discrete Applied Mathematics, vol. 158, no. 15, pp. 1571-1578, 2010.

[14] A. Ilić and B. Zhou, "On reformulated Zagreb indices," Discrete Applied Mathematics, vol. 160, pp. 204-209, 2012.

[15] A. Miličević, S. Nikolić, and N. Trinajstić, "On reformulated Zagreb indices,” Molecular Diversity, vol. 8, pp. 393-399, 2004.

[16] B. Basavanagoud and S. Patil, "A note on hyper-Zagreb index of graph operations," Iranian Journal of Mathematical Chemistry, vol. 7, pp. 89-92, 2016.

[17] K. Pattabiraman and M. Vijayaragavan, "Hyper Zagreb indices and its coindices of graphs," Bulletin of the International Mathematical Virtual Institute, vol. 7, pp. 31-41, 2017.

[18] I. Gutman, "Multiplicative Zagreb indices of trees," Bulletin of the International Mathematical Virtual Institute, vol. 1, pp. 13-19, 2011.

[19] K. Xu and H. Hua, "A unified approach to extremal multiplicative Zagreb indices for trees, unicyclic and bicyclic graphs," MATCH Communications in Mathematical and in Computer Chemistry, vol. 68, pp. 241-256, 2012.

[20] I. Gutman, N. Dehgardi, and H. Aram, "On general first Zagreb index of graphs with fixed maximum degree," Bulletin of the International Mathematical Virtual Institute, vol. 6, pp. 251-258, 2016.

[21] M. Eliasi, A. Iranmanesh, and I. Gutman, "Multiplicative versions of first Zagreb index," MATCH Communications in Mathematical and in Computer Chemistry, vol. 68, pp. 217230, 2012.

[22] K. Xu and K. C. Das, "Trees, unicyclic, and bicyclic graphs extremal with respect to multiplicative sum Zagreb index," MATCH Communications in Mathematical and in Computer Chemistry, vol. 68, pp. 257-272, 2012.

[23] K. Xu, K. C. Das, and K. Tang, "On the multiplicative Zagreb coindex of graphs," Opuscula Mathematica, vol. 33, pp. 191204, 2013.

[24] L. Bedratyuk and O. Savenko, "The star sequence and the general first Zagreb index," MATCH Communications in Mathematical and in Computer Chemistry, vol. 79, pp. 407414, 2018.

[25] F. Zhan, Y. Qiao, and J. Cai, "Relations between the first Zagreb index and spectral moment of graphs," MATCH Communications in Mathematical and in Computer Chemistry, vol. 81, pp. 383-392, 2019.

[26] R. Rasi, S. M. Sheikholeslami, and A. Behmaram, "An upper bound on the first Zagreb index and coindex in trees," Iranian Journal of Mathematical Chemistry, vol. 8, pp. 71-82, 2017.

[27] A. Behtoei, "Some relations and bounds for the general first Zagreb index," MATCH Communications in Mathematical and in Computer Chemistry, vol. 81, pp. 361-370, 2019.

[28] Y. Ma, S. Cao, Y. Shi, I. Gutman, M. Dehmer, and B. Furtula, "From the connectivity index to various Randić-type descriptors," MATCH Communications in Mathematical and in Computer Chemistry, vol. 80, pp. 85-106, 2018.

[29] D. Zhao, Z. Iqbal, R. Irfan et al., "Comparison of irregularity indices of several dendrimers structures," Processes, vol. 7, no. 10, pp. 1-14, 2019.
[30] A. Alwardi, A. Alqesmah, R. Rangarajan, and I. Naci Cangul, "Entire Zagreb indices of graphs," Discrete Mathematics, Algorithms and Applications, vol. 10, no. 16, p. 1850037, 2018.

[31] A. Ghalavand and A. R. Ashrafi, "Bounds on the entire Zagreb indices of graphs," MATCH Communications in Mathematical and in Computer Chemistry, vol. 81, pp. 371-381, 2019. 